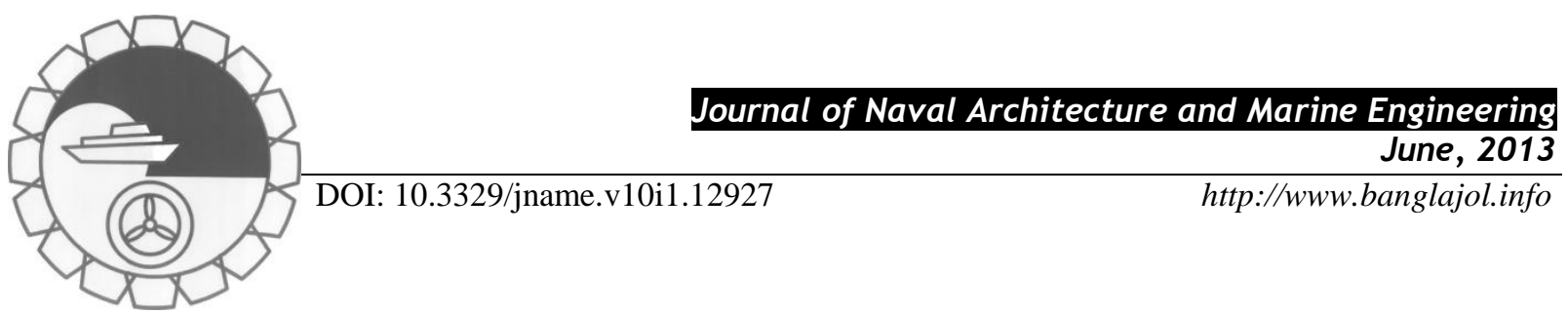

\title{
AN EVOLUTIONARY OPTIMIZATION TECHNIQUE APPLIED TO RESISTANCE REDUCTION OF THE SHIP HULL FORM Hassan Zakerdoost ${ }^{1}$, Hassan Ghassemi ${ }^{2}$ and Mahmoud Ghiasi ${ }^{3}$
}

Department of Ocean Engineering, Amirkabir University of Technology, Tehran, IRAN

Email: $\underline{1}$ h.zakerdoost@aut.ac.ir, ${ }^{2}$ gasemi@aut.ac.ir and ${ }^{3}$ mghiasi@aut.ac.ir

\begin{abstract}
:
Hull form optimization from a hydrodynamic performance point of view is an important aspect of ship design. This paper presents a computational method to estimate the ship resistance (viscous and wave) in calm-water. In the optimization process the evolution strategy (ES) technique is linked to the computational method to obtain an optimum hull form by taking into account the displacement as design constraint. For allowing the large variation of hull form during optimization process the hull surface is represented by NURBS. New hull forms are obtained from the well-known S60 hull and the classical Wigley hull taken as initial hulls in the optimization process at Fn=0.316. The optimization variables are a combination of ship hull offsets and main dimensions. The benchmark results for two test cases indicate that the total resistance of optimized hulls is reduced significantly..
\end{abstract}

Keywords: Hydrodynamics, optimization, hull form, evolution strategies, calm-water resistance.

\section{NOMENCLATURE}

\section{$F n$}

$f_{i}(\bar{x})$

$\bar{x}$

$\mathrm{m}$

$q$

$p$

$S$

$\mathcal{F}$

$h_{i}(\bar{x})$

$g_{i}(\bar{x})$

$x_{i}$

$C_{T} \quad$ total resistance coefficient

$\mathrm{C}_{\mathrm{f}} \quad$ frictional resistance coefficient

$\mathrm{C}_{\mathrm{W}} \quad$ wave resistance coefficient

$k \quad$ form factor

$R n \quad$ Reynolds number

$U \quad$ ship speed

$L \quad$ ship length
$B \quad$ ship width

$D \quad$ ship draft

$P_{i, j} \quad$ bidirectional control net

$w_{i, j} \quad$ weights

$N_{i, p}(u), N_{j, q}(v)$ NURB-Spline basis functions

$u, v \quad$ directions of surface

$g$ gravity acceleration

$N_{i}(0,1)$ random number

$\mathbb{R}^{n} \quad$ domains of variables

\section{Greek symbols}

$\sigma_{i} \quad$ mutation step size

$\mu \quad$ number of parents

$\lambda$ number of offspring

$v \quad$ kinematic viscosity

$\tau, \eta \quad$ learning rates

\section{Introduction}

There are several significant performances that have to be taken into account in the ship design. From a hydrodynamic performance point of view the ship resistance is one of the most important performances in the ship hull form optimization. Since small changes in the ship resistance can lead to significant changes in fuel consumption and thus ship cost. It is worth noting that for a comprehensive and detailed ship hydrodynamic optimization all objective functions such as resistance, stability, sea-keeping etc. must be considered, because it is clear that consideration of an objective function without the other ones gives unrealistic and impractical results.

Some researchers have considered two or three objective functions for optimizing hull form and some others only one objective function. For example Gammon (2011) uses three objective functions in his study, Biliotti et 
al. (2011) and Grigoropoulos and Chalkias (2010) utilize two objective functions in their work and many researchers use only one objective function (Han et al., 2012 and Kim et al.., 2012).

Zhang (2009 and 2012), Kim et al. (2009 and 2008) and Saha et al. (2004) employed different types of the Nonlinear programming (NLP) as optimization techniques. However at present evolutionary Algorithms (EAs) such as evolution strategies (ESs) and evolutionary programming (EP) are most widely used in hull shape modification. The ES employed in this work is significantly faster at numerical optimization than traditional GA and also more likely to find a function's true global extremum. Jun and Kuniharu (2004) presented a singleobjective optimization algorithm based on genetic algorithm to improve hull form of a catamaran. In another study a hull form with respect to seakeeping and total resistance as objective functions is optimized by using evolutionary strategies (Grigoropoulos et al., 2004).

A well-known theoretical method to compute the wave making resistance is Michell's thin ship theory. Gotman (2002) conducted a systematical study of Michell's integral and an investigation into a difference between the linear theory and experiment. Genetic algorithm technique is employed by Karim et al. (2004) to optimize the hydrofoil and propeller incorporating potential based boundary element method. A method based on linear thinship theory to calculate the wave wake generated by a ship is presented by Day and Doctors (2001). They used the elemental tent functions as building blocks to represent the hull. The CFD methods such as Rankine source panel method (Vyselaar et al., 2007) have several disadvantages compared to Michell's integral include excessive computational requirements and preprocessing discretization requirements that are too expensive for routine applications such as ship hull form optimization. De and Kumar (2006) described and evaluated a scheme of engineering-economic analysis for determining optimum ship's main dimensions and power requirement at basic design stage.

Evolutionary algorithms operate on populations. Each individual in the population denotes a search point in the space of potential solutions to a given problem. The population moves toward regions of the search space with high quality. The benefits of evolutionary algorithms are: widely applicable, no presumptions with respect to problem space, easy to incorporate other methods, low development and application costs, can be run interactively, solutions are interpretable, provide many alternative solutions and intrinsic parallelism, straightforward parallel implementations, accommodate user proposed solutions. The appropriate problems for EAs are complex problems with one or more of the following features: complex relationships between parameters, many free parameters, many local optima, mixed types of parameters (integer, real), multiple objectives, changing conditions (dynamic fitness landscape), noisy data.

Application of the evolution strategy as an optimization methodology in the field of the hydrodynamic optimization of ship hull form is very limited. Rechenberg (1973), Schwefel (1995), Beyer and Schwefel (2002) who were working on an application concerning shape optimization of a bent pipe and a flashing nozzle introduced the evolution strategies in the early 1960 s.

In this study, after problem formulation and especially the explanation of linearized thin ship theory and a particular form of the optimization algorithm (evolution strategy), results of application of this methodology using two different cases of the Wigley hull and the S60 hull are presented, and in both ones allowing principal parameters of length, beam and draft to change simultaneously with the offset of hull surface.

\section{Formulation of Optimization Problem and the Evolution Strategy}

The standard mathematical form of a constrained optimization problem is as follows:

Optimize $F(\bar{x})=\left[f_{1}(x), f_{2}(x), \ldots, f_{m}(x)\right] \quad x \in \mathrm{R}^{\mathrm{n}}$

Subject to

$$
\begin{aligned}
& h_{i}(\bar{x})=0 \quad i=1, \ldots \ldots \ldots, q \\
& g_{i}(\bar{x}) \leq 0 \quad i=1, \ldots \ldots \ldots, p
\end{aligned}
$$

Where $f_{i}(\bar{x})$ is the objective function, $m$ is the number of objective function, $q$ is the number of equality constraints, $p$ is the number of inequality constraints and $\bar{x}=\left(x_{1}, \ldots, x_{n}\right) \in \mathcal{F} \subseteq S$ is a solution or individual. The set $S \subseteq \mathbb{R}^{n}$ defines the search space and the set $\mathcal{F} \subseteq \mathrm{S}$ defines a feasible search space. The search space $\mathrm{S}$ is defined as an n-dimensional rectangle in $\mathbb{R}^{n}$ (domains of variables defined by their lower and upper bounds):

$$
l(i) \leq x_{i} \leq u(i) \quad 1 \leq i \leq n
$$


The constraints define the feasible area. This means that if the design variables vector $\bar{x}$ be in agreement with all constraints $h_{i}(\bar{x})$ (equality constraint) and $g_{i}(\bar{x})$ (inequality constraint), it belongs to the feasible area.

In this study design variables vector include the main parameters (length, beam, draft) and the hull control points which are limited by the lower and upper bounds. The ship hull displacement also is an equality constraint.

The basis of ESs that are the most popular algorithms for solving continuous optimization problems is similar to other evolutionary algorithms: consider a population of individuals; the environmental pressure causes natural selection which leads to an increase in the fitness of the population.

Consider an evaluation function to be minimized. A set of candidate solutions can be randomly generated and the objective function can be used as a measure of how individuals have performed in the problem domain (an abstract fitness measure) - the lower the better. According to this fitness, some of the better solutions are selected to seed the next generation by applying recombination and/or mutation operators to them. The recombination (also called crossover) operator is used to generate new candidate solutions (offspring) from existing ones, they take two or more selected candidates (parents) from the population pool and exchange some parts of the solutions to form one or more offspring. Mutation operator is used to generate one offspring from one parent by changing some parts of the candidate solution. Applying recombination and mutation operators causes a set of new candidates (offspring) competing based on their fitness (and possibly age) with the old candidates (the parents) for a place in the next generation. This procedure can be iterated until a solution with sufficiently quality (fitness) is found or a previously set computational time limit is reached. In other words, the end conditions must be satisfied. The composed application of selection and variation operators (recombination and mutation) improves fitness values in consecutive population.

Variables in evolution strategies are divided into two categories: object and strategy variables. Standard representation of variables in evolution strategies is as real-valued vectors because ESs is usually used for continuous parameters. A form of an individual in ESs is as follows:

$$
<x_{1}, \ldots, x_{n}, \sigma_{1}, \ldots, \sigma_{n_{\sigma}}>
$$

where $x_{i}$ is the object variable and $\sigma_{i}$ is mutation step size or standard deviation (strategy variable). A normal (Gaussian) distribution with zero mean and standard deviation $\sigma$ in the mutation operator of ES is a major characteristic. A common approach of mutation operator is uncorrelated mutation with $\mathrm{n}$ step sizes. The advantage of this approach is that the fitness surface can be treated in different directions with different slopes. The mutation methodology for $i \in\{1, \ldots, n\}$ is as follows:

$$
\begin{aligned}
& \sigma_{i}^{t+1}=\sigma_{i}^{t} \cdot e^{\tau \cdot N(0,1)+\eta \cdot N_{i}(0,1)} \\
& x_{i}^{t+1}=x_{i}^{t}+\sigma_{i}^{t+1} \cdot N_{i}(0,1)
\end{aligned}
$$

where $\tau \propto 1 / \sqrt{2 n}, \eta \propto 1 / \sqrt{2 \sqrt{n}}$ and $N_{i}(0,1)$ are random numbers drawn from the standard normal distribution. Note that $N(0,1)$ is drawn only once (Back et al., 2000). Global intermediate recombination and global discrete recombination are typically two main types of recombination used in ES. One child $\bar{z}$ is produced from two parents $\overline{\mathrm{x}}$ and $\overline{\mathrm{y}}$ drawn randomly from $\mu$ parents for each position $\mathrm{i} \in\{1, \ldots, \mathrm{n}\}$ where

$$
z_{i}=\left\{\begin{array}{l}
\left(x_{i}+y_{i}\right) / 2 \\
x_{i} \text { or } y_{i} \text { chosen randomly }
\end{array}\right.
$$

\section{global intermediate recombination global discrete recombination}

The global intermediate recombination and the global discrete recombination are preferred for use in the strategy variables and the object variables respectively. The $(\mu, \lambda)$ survivor selection scheme has advantages over its competitor, the $(\mu+\lambda)$ selection scheme but the $(\mu+\lambda)$ selection scheme is an elitist mechanism that can maintain the best solution to each generation (Eiben and Smith, 2003). The self-adaptation of the standard deviation of the Gaussian distribution used in the mutation operator is the special characteristic of ESs (Back and Schwefel, 1993). Moreover, the ES can locate global optimum and escape from local optimums, can locate feasible optimal solution in constrained optimization problems and use the values and parameters themselves, not a coding of them. 


\section{Resistance Calculation}

The ship is assumed as a rigid floating body that moves with constant velocity relative to the fluid. The total calm water resistance is composed of the viscous resistance and the wave resistance. The total resistance coefficient is calculated as follows:

$$
C_{T}=(1+k) C_{f}+C_{W}
$$

where $C_{f}$ is the frictional resistance coefficient, $C_{W}$ is the wave resistance coefficient and $k$ is the form factor given by Percival et al. (2001) as:

$$
k=0.6 \sqrt{\nabla / L^{3}}+9 \nabla / L^{3}, \quad 0.05 \leq k \leq 0.4
$$

The international towing tank conference that is held in Madrid in 1957 (ITTC 57) introduced the following relationship as ship-model correlation line for calculating the frictional resistance coefficient:

$$
C_{f}=\frac{0.075}{\left(\log _{10} R n-2\right)^{2}}
$$

where $R n$ is the Reynolds number given by

$$
R n=\frac{U L}{v}
$$

where $U$ is the ship speed, $L$ is the ship length and $v$ is the kinematic viscosity.

There are some theories to determine the wave-making resistance like Michell's theory using potential flow as described by Tuck and Lazauskas (1998). The Michell's Integral used in this study includes only the transom stern and the offsets of bow are assumed to be zero, otherwise will be added a complexity to the integral. The wave resistance coefficient follows by normalization according to:

$$
C_{W}=\frac{R_{w}}{0.5 \rho S U^{2}}
$$

where $S$ denoting the (static) wetted surface area. The comparison of Ju's experiment (Ju, 1983) and Insel's experiment (Insel, 1990) with a $3.048 \mathrm{~m}$ and a $1.8 \mathrm{~m}$ Wigley hulls with length to beam ratio $L / B=10$ and length to draft ratio $L / T=16$ respectively are shown in Figure 1. Using the numerical method described above for computing total resistance leads to good agreement and errors between predictions and the experiments lie within about $10 \%$ for the entire range of Froude number. The total resistance (objective function) is a function of the main dimension (length, beam and draft) and the hull offsets of the ship in the optimization process which must be minimized. It's integrand is highly oscillatory, and special techniques are needed to evaluate the integrals. We use Filon's quadrature (Davis and Rabinowitz, 1984) to capture the rapid oscillations as $|\theta| \rightarrow$ $\pi / 2$. Conventional quadratures fail to capture the correct decay of the spectrum in this region (Tuck et al., 2002).
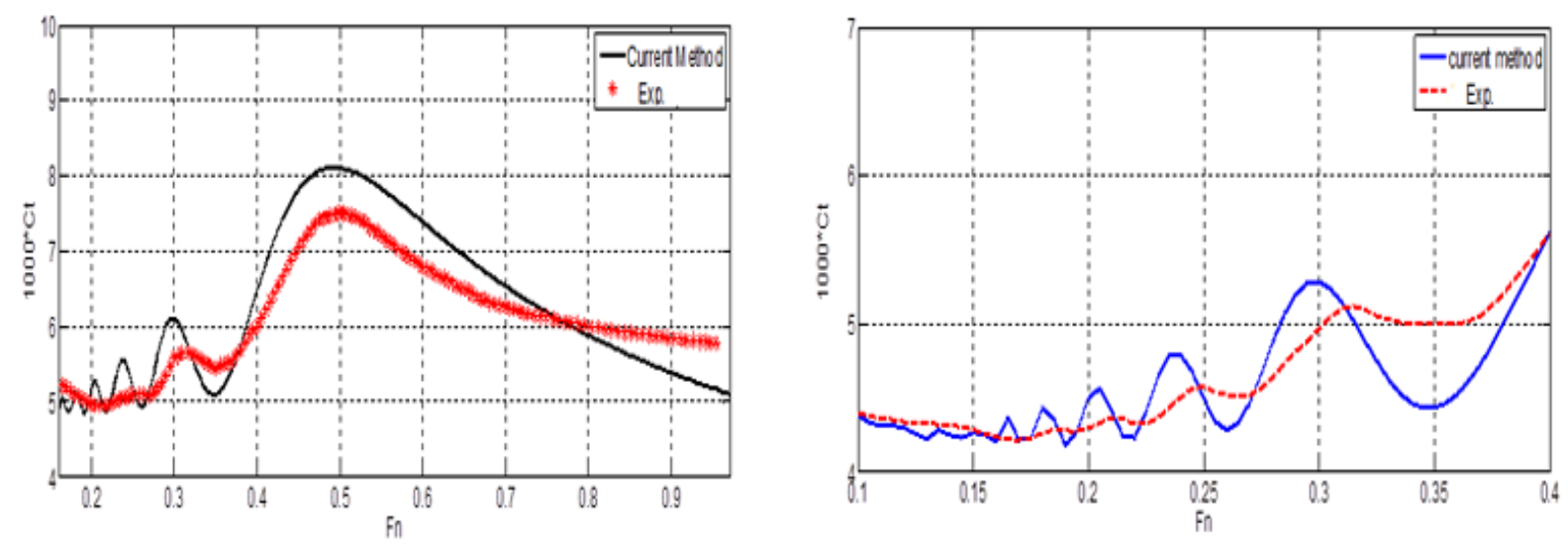

a) $\mathrm{L}=1.8[\mathrm{~m}]$,

b) $\mathrm{L}=3.048[\mathrm{~m}]$

Figure 1: Comparison of total resistance coefficient for two models of the Wigley hull

\section{Procedure of the Hull Form Optimization}

The numerical optimization can be conducted by iterative evaluations of the total resistance function as the objective function. Each chromosome (biologic name of a solution) in the optimization algorithm consists of control points of ship hull, length (waterline length), beam (in waterline) and draft. Because of large number of 
variables, the evolution strategy is chosen as a successful algorithm for our work. During optimization the ship displacement as design equality constraint is kept unchanged. In order to restrict the search space and to keep the optimal hull near the initial hull for comparison, the length, beam and draft are limited to a $\pm 20 \%$ variation in the main dimensions and the offsets points are limited to $\pm 6 \%$ of the original hull offsets for the cases in which only the offset values are changed and $\pm 2 \%$ of the initial hull offsets for the cases in which both the offset values and the principal dimensions are altered.

The modeling of ship hull form is based on NURBS (Non Uniform Rational B-Splines) (Piegl and Tiller, 1995) for allowing the large variation of hull form during optimization process. A NURBS surface of degree $p$ in the $u$ direction and degree $\mathrm{q}$ in the $\mathrm{v}$ direction is a bi-variate vector-valued piecewise rational function of the form

$$
S(u, v)=\left(\begin{array}{c}
x(u, v) \\
y(u, v) \\
z(u, v)
\end{array}\right)=\frac{\sum_{i=0}^{n} \sum_{j=0}^{m} N_{i, p}(u) N_{j, q}(v) w_{i, j} P_{i, j}}{\sum_{i=0}^{n} \sum_{j=0}^{m} N_{i, p}(u) N_{j, q}(v) w_{i, j}} 0 \leq u, v \leq 1
$$

The $P_{i, j}$ form a bidirectional control net, $w_{i, j}$ are the weights, and $N_{i, p}(u)$ and $N_{j, q}(v)$ are the non-rational B-Spline basis functions defined on the knot vectors. In this study the cubic surface $(\mathrm{p}=\mathrm{q}=3)$ is used and the control points $\left(P_{i, j}\right)$ 'consequently hull offsets' with/without principal dimensions are design variables of the constrained optimization problem.

The Wigley model and the S60 model are two popular models in ship hydrodynamics experiments that are used in many studies as the test models. We employed these models to compare numerical results. The standard Wigley hull is a mathematical displacement hull form, the geometric surface of which can be defined as

$$
f(x, y)=\frac{B}{2}\left[1-\left(\frac{2 x}{L}\right)^{2}\right]\left[1-\left(\frac{z}{T}\right)^{2}\right]
$$

The total resistance may be computed from equation (3). The hull form optimization is carried out at a single Froude number $(F n=U / \sqrt{g L})$ of 0.316 where $U$ and $L$ is constant speed and waterline length of the model respectively. Table 1 shows the main characteristics of the Wigley and S60 hull models.

Table 1: Main characteristics of the Wigley and S60 hull models

\begin{tabular}{|l|l|l|l|l|l|}
\hline Parameters & Length $(\mathrm{m})$ & Beam $(\mathrm{m})$ & Draft $(\mathrm{m})$ & $\begin{array}{l}\text { Wetted hull } \\
\text { surface }\left(\mathrm{m}^{2}\right)\end{array}$ & Design Fn \\
\cline { 1 - 5 } Model type & & & & & \\
\hline Wigley hull form & 1.8 & 0.18 & 0.1125 & 1.383 & 0.316 \\
\hline S60 hull form & 4.689 & 0.6252 & 0.25 & 2.91 & 0.316 \\
\hline
\end{tabular}

The numerical optimization is conducted by the evolution strategy algorithm. The control points of hull surface and principal dimensions can be represented by real-valued vectors in the limits as already mentioned. The global intermediate recombination and the global discrete recombination have been used in the strategy variables and the object variables respectively. The mutation operator with $n$ step size (using Gaussian distribution) has been applied to the candidate solution. The recombination rate has been 0.80 , while the mutation rate has been 1.0 per one individual. The parent selection has been performed by a uniform random distribution. According to results of tests carried out by authors the $(\mu, \lambda)$ scheme and the $(\mu+\lambda)$ scheme has been selected as appropriate survivor selection mechanisms for the first and second test cases employed the Wigley hull and the S60 hull as mother models. The program code has run in a system with $2.2 \mathrm{GHz}$ processor.

\section{Results and Discussion}

5.1. Case study of the Wigley hull

In order to perform the optimization of hull for minimizing the total resistance of the ship, which is a key factor in the hydrodynamic design of hull, and to determine the preliminary design parameters to satisfy the design requirements given by the owner or client, it is necessary the candidate solutions generated are permitted to vary by changing the control points of the hull form and the main dimensions. The first example is for the 
hydrodynamic optimization of the Wigley hull form with respect to the minimum total resistance. The Wigley model with length to beam ratio $L / B=10$ and length to draft ratio $L / T=16$ is optimized for a single speed, corresponding to Froude number of 0.316 . The offsets values and main dimensions of the hull are limited in the range of 98 to 102 and 80 to 120 percent of initial ones respectively. The number 280 hull forms in each generation are created and among them, the best 40 hull forms are selected to seed the next generation based on the fitness i.e. the lower total resistance the better hull form. Figure 2 depicts body-plan of the optimal hull form generated by use of the evolution strategy optimization technique and body plan of the initial Wigley hull. The single-speed optimization procedure improved the initial hull and produced a reasonable hull form. The new hull is smoothed because of using NURBS.

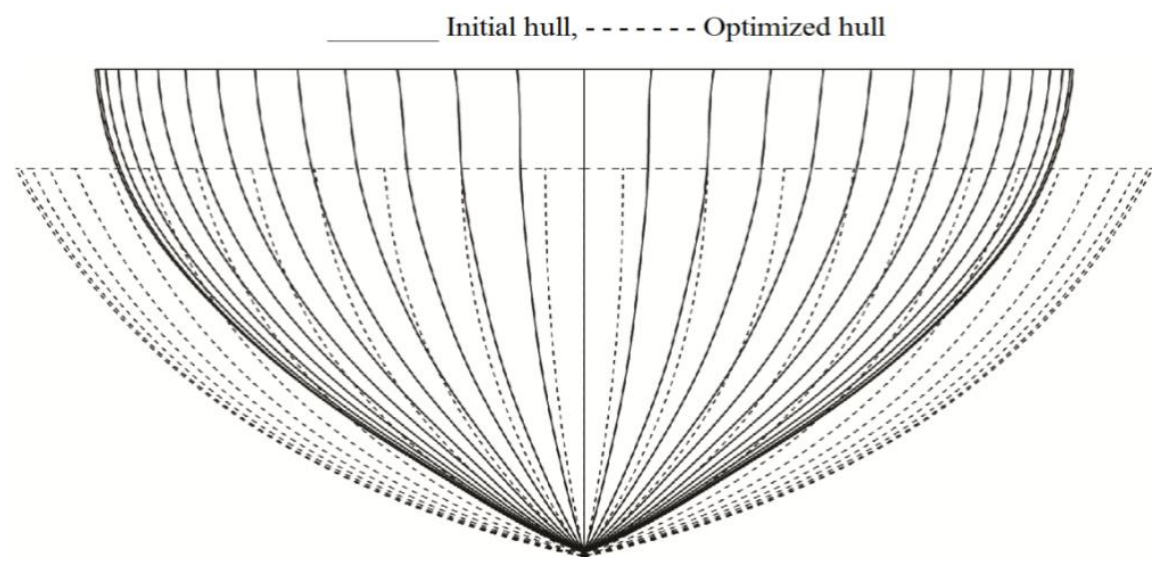

Figure 2: Body plans of original Wigley hull and optimal hull

As can be seen in Figure 2, the beam of the optimized hull is wider than the beam of the initial Wigley hull and the draft of the optimized hull has decreased dramatically. The 3D and hull renderings of the initial and optimized Wigley hull form are presented in Figure 3. During the run of the optimization algorithm in addition to the hull offsets the length, beam and draft of the hull are changed. The variation of the main dimensions and the wetted surface of the hull versus evaluation number are shown in Figure 4. These two figures (3 and 4 ) confirm that the hull has a tendency toward a wider beam and shallower draft during the optimization algorithm. The length of the hull is rapidly decreased in the initial evaluations and after that is remained fixed. The changes in the variable parameters of the hull are to reach minimum resistance and match the constraint for the displacement.

The changes of three non-dimensional parameters, i.e., frictional resistance, wave resistance and total resistance versus evaluation number of the objective function are shown in Figure 5. In all figures, $R_{t 0}$ is the total resistance of the initial hull also $R_{f}, R_{w}$ and $R_{t}$ are the frictional resistance, the wave resistance and the total resistance of the hull respectively that has being optimized. The reduction percent of the wave resistance is more than the reduction percent of the frictional resistance; this is because of the greater impact of the hull geometry change on the wave resistance than the frictional resistance.

The block coefficient of the optimized hull also is increased about 20\%. Figure 6 shows comparison of the total resistance for the optimal hull and the initial Wigley hull within a speed range. As can be seen in this figure although the hull form has been optimized for a single speed $(F n=0.316$ or $V=1.33)$, the total resistance of the optimal hull form is less over the broad range of the speed. As the speed increases, the difference between the total resistances of original and optimized hull forms (resistance reduction) increases. The optimization takes around 85 minutes. 


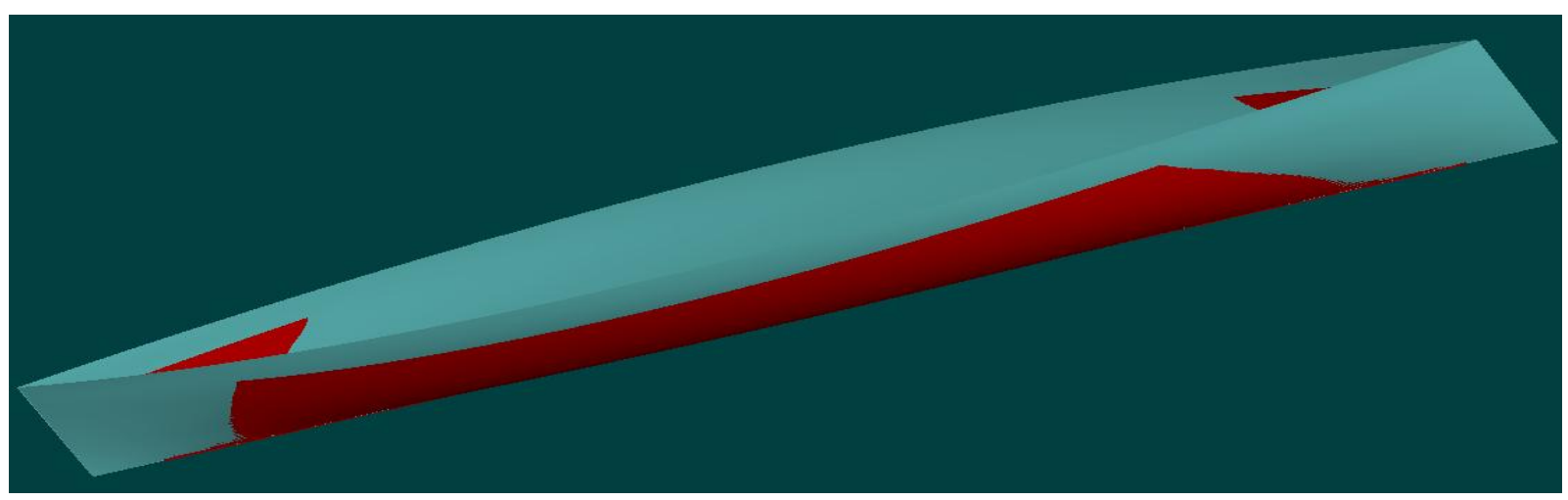

3D hull form, (Red is optimized hull and blue is initial hull)

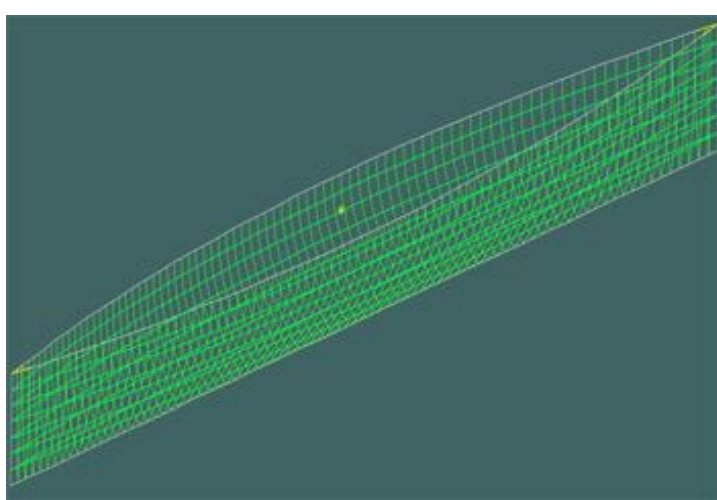

initial hull

Figure 3: Wigley hull form rendering

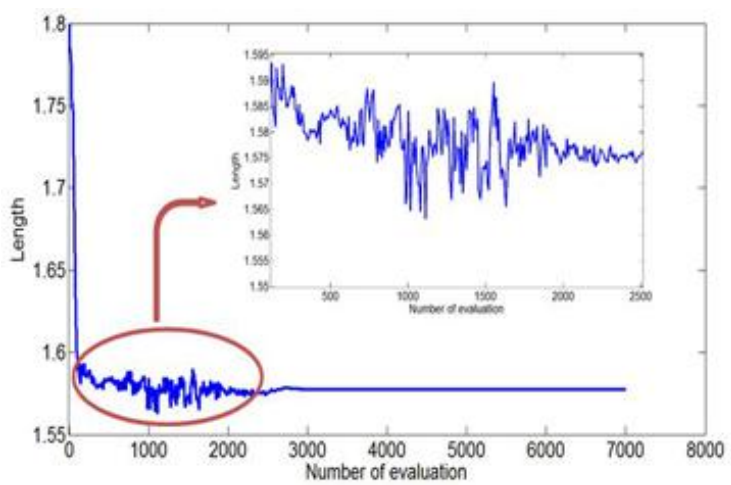

a) length

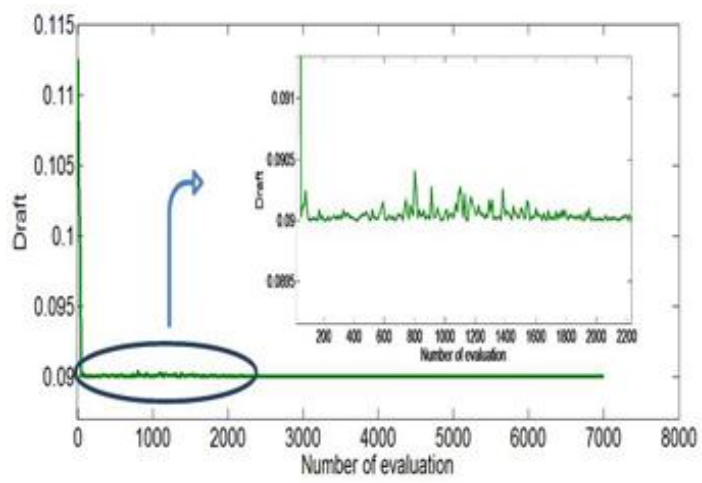

c) draft

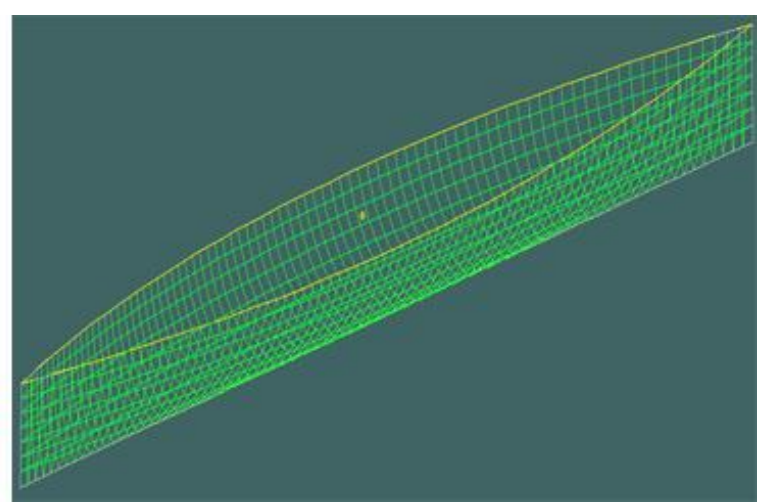

optimized hull

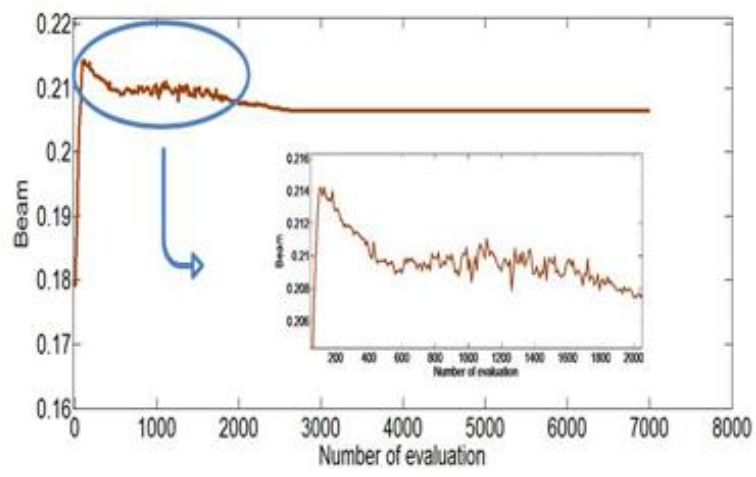

b) beam

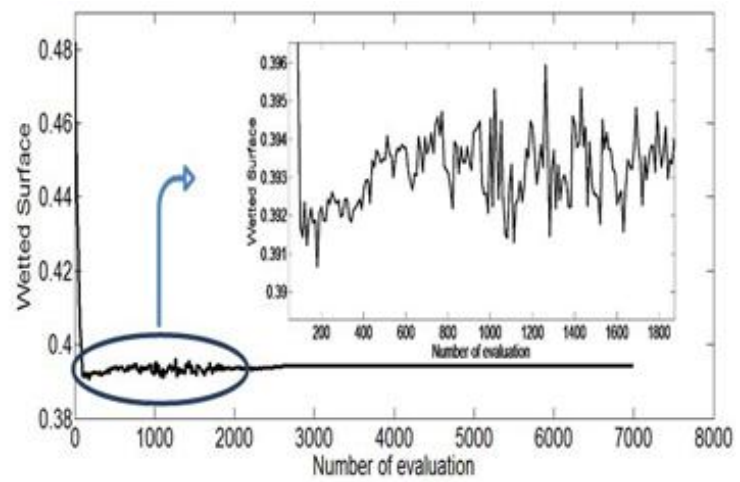

d) wetted surface

Figure 4: Variation of dimensions parameters of the hull 


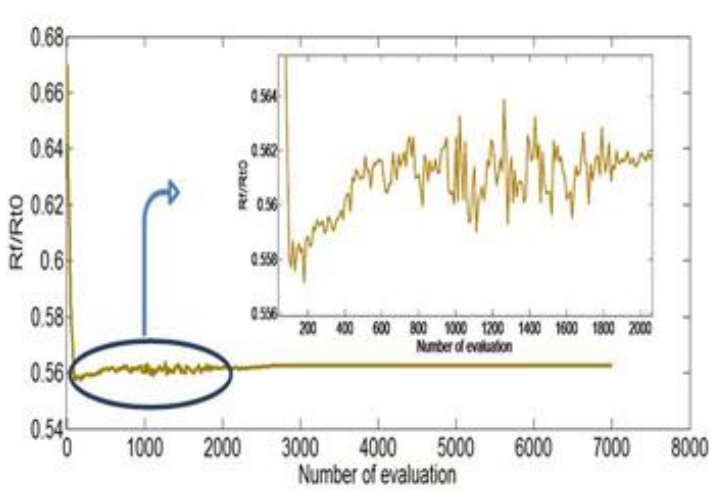

a) frictional resistance

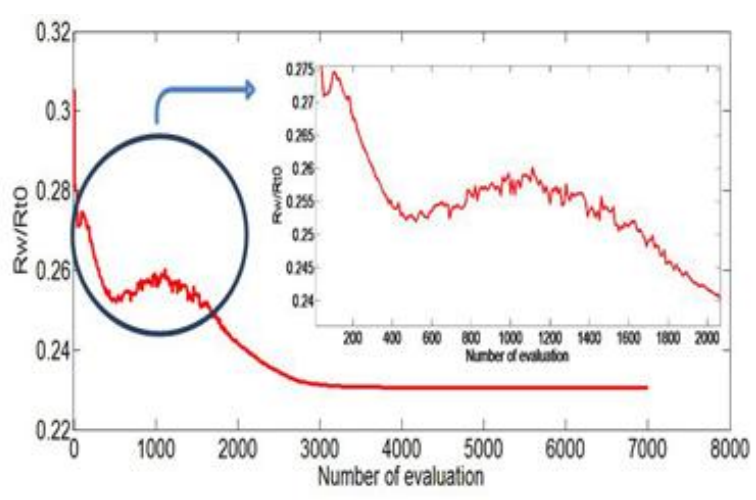

b) wave resistance

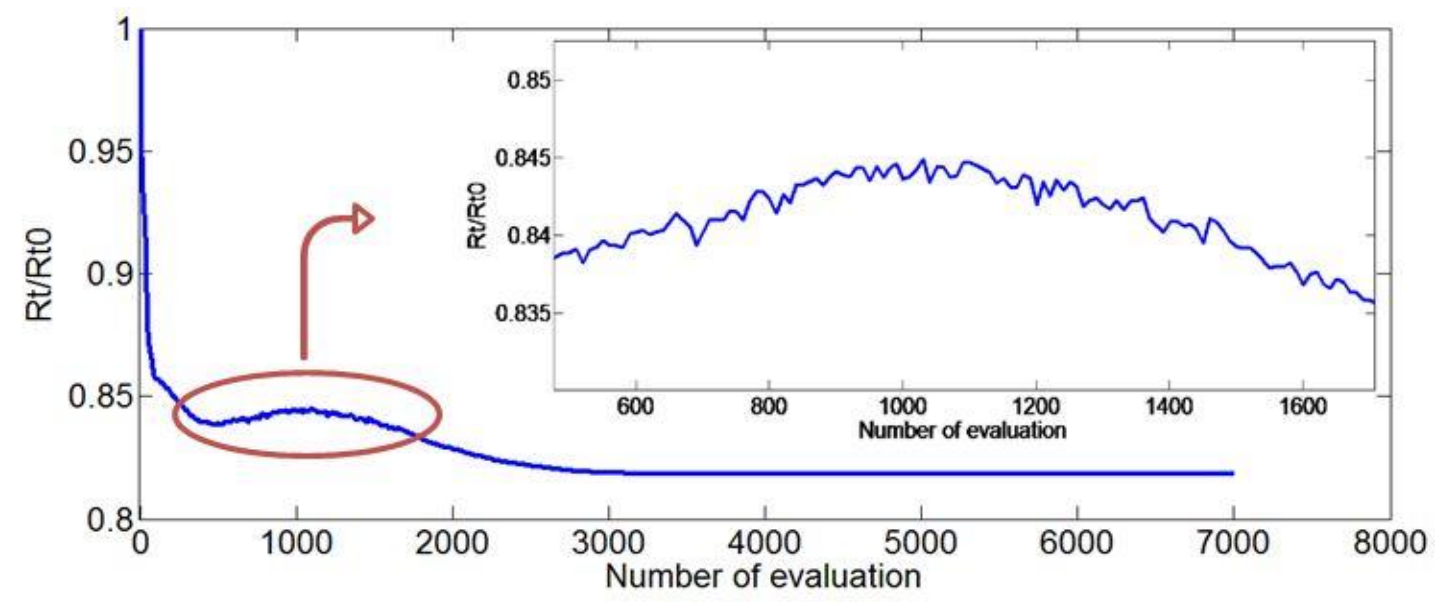

c) total resistance

Figure 5: The history of the resistance convergence of the Wigley hull by evaluation number

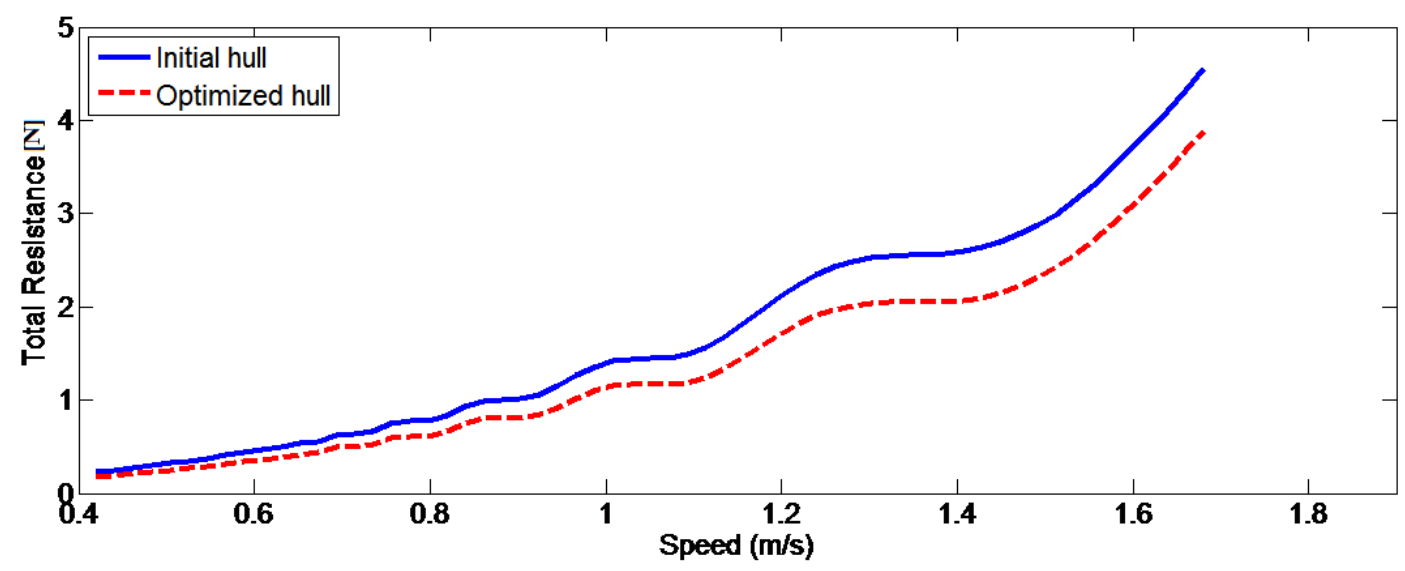

Figure 6: The total resistance of the initial Wigley hull and the optimized hull.

\subsection{Case study of the $\mathrm{S} 60$ hull}

In this example, a $4.689 \mathrm{~m}$ in length of the $\mathrm{S} 60$ hull form $\left(C_{B}=0.6\right)$ with length to beam ratio $L / B=7.5$ and beam to draft ratio $B / T=2.5$ is chosen in order to derive a hull with minimum resistance at $F n=0.316$. The water is calm and deep with infinite bounds. As was said before the optimization of the hull form is based on minimizing the main hydrodynamic factor of the ship i.e. total resistance with given design constraints. The variation range in the offsets values is between 98 and 102 percent of the initial S60 hull offsets and the main dimensions are changed in the limits between 80 and 120 percent of the main dimensions of the initial hull. The 
body-plan of the initial hull form (Continuous lines) and the optimized hull form (dashed lines) are shown in Figure 7. In each generation 154 hulls are created and then among them, the best 22 hull forms are considered to go to the next generation based on lower resistance. Use of the evolution strategy algorithm in combination with the NURBS produced optimized fair hull. To acquire a hull form with minimum resistance and match the constraint for the displacement, the beam and draft of the optimized hull are wider and shallower than the initial S60 hull.

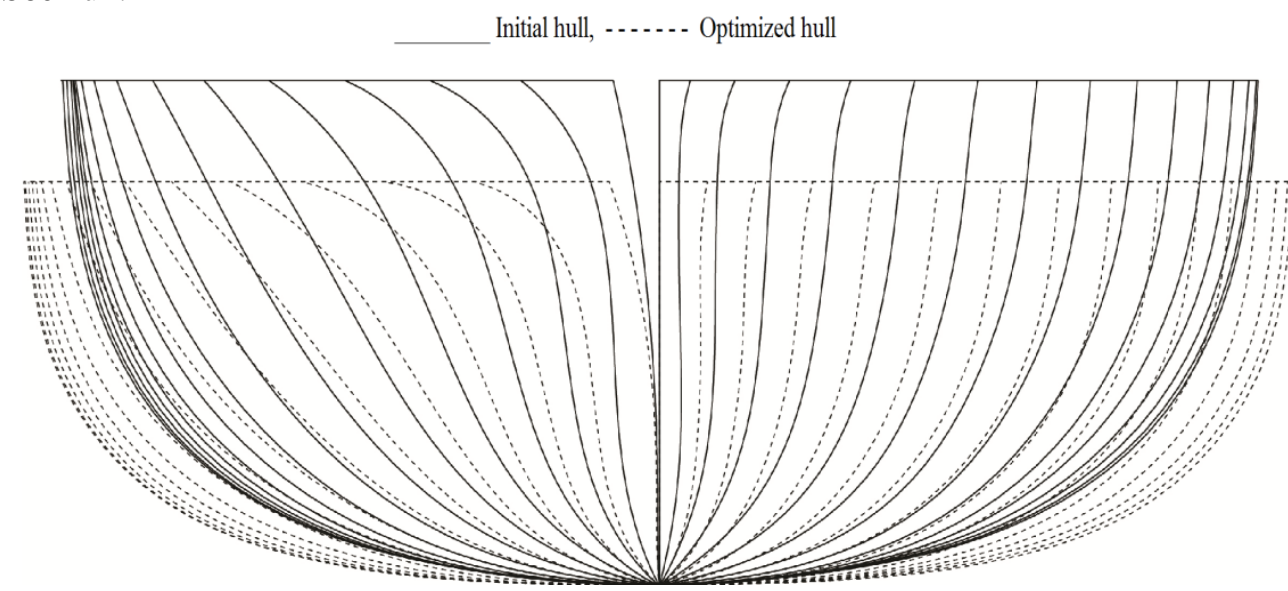

Figure 7: Body plans of original S60 $\left(C_{B}=0.6\right)$ hull and optimal hull

The 3D and hull rendering of the initial and optimized S60 hull form is shown in Figure 8. During the implementation of the optimization algorithm in addition to hull offsets the length, beam and draft of the hull are varied and the changes of them and wetted surface of the hull by evaluation number are as in the previous case, but the difference is the hull has a tendency towards approximately longer length (see Figures 7 and 8 ). As can be seen in these figures, the significant changes of four characteristics of the hull are at the early evaluation of objective function and after that remain fixed.

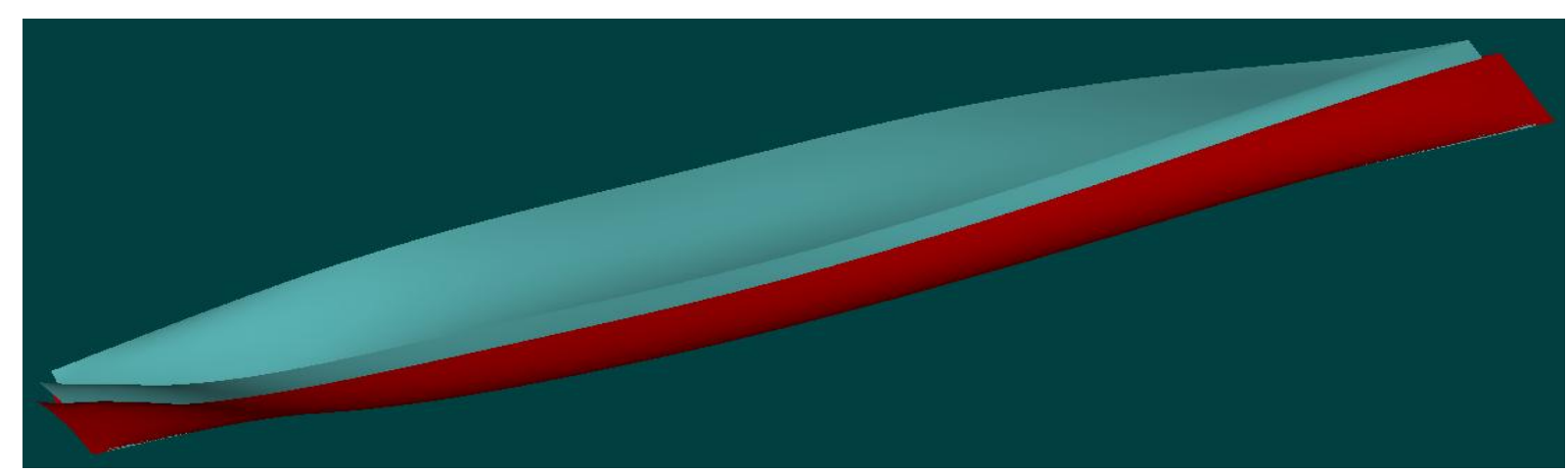

3D hull form, (Red is optimized hull and blue is initial hull)

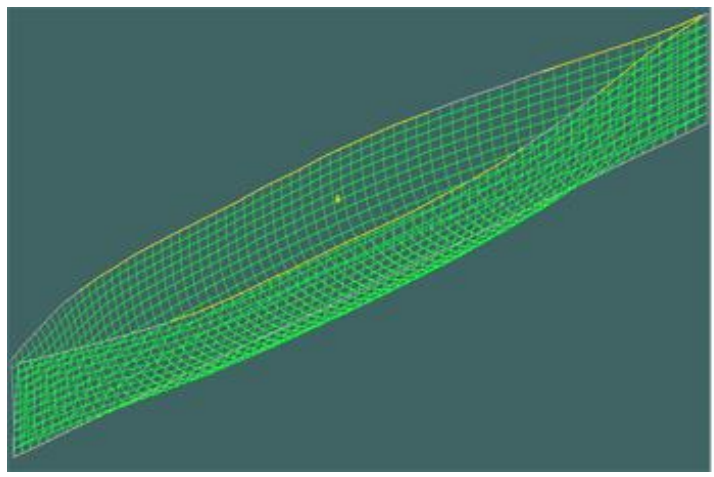

Initial hull

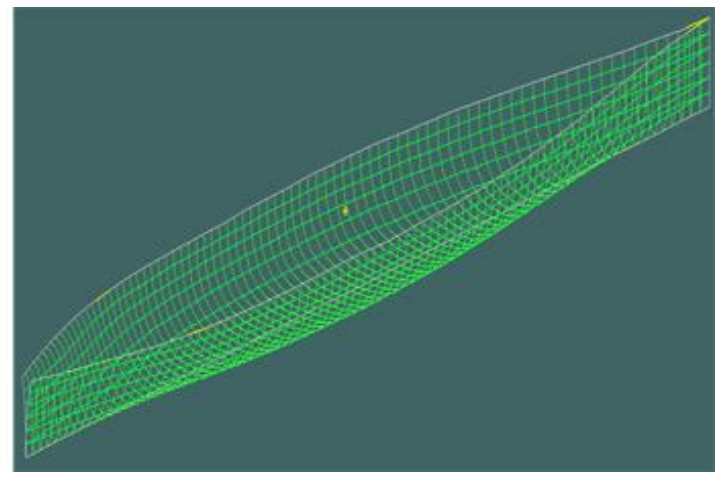

Optimized hull

Figure 8: S60 hull form renderings 


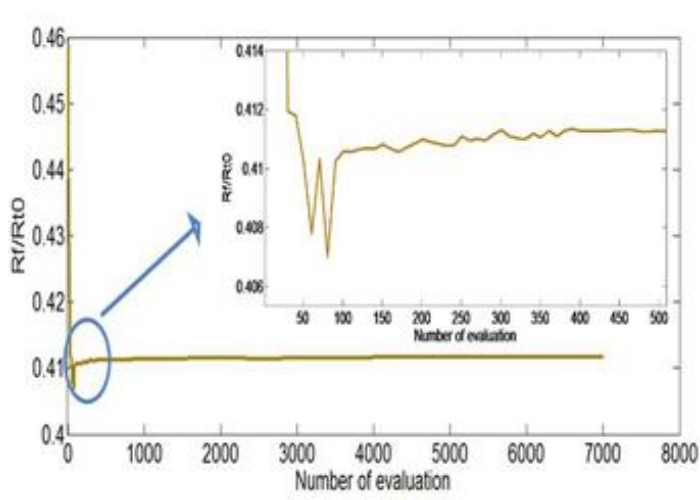

a) frictional resistance

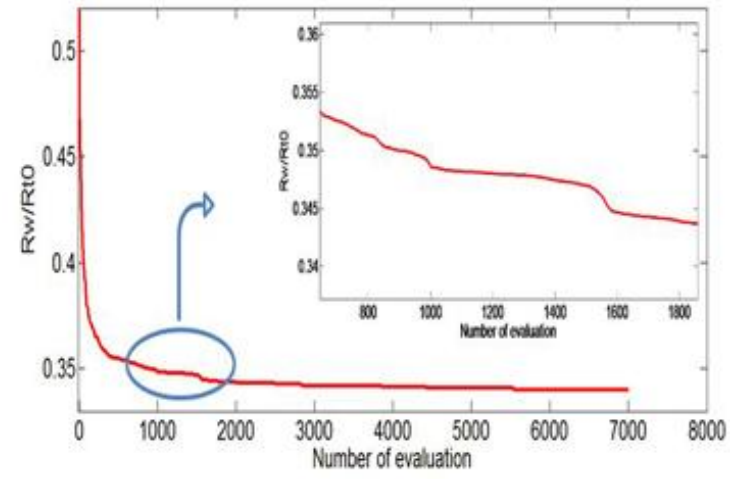

b) wave resistance

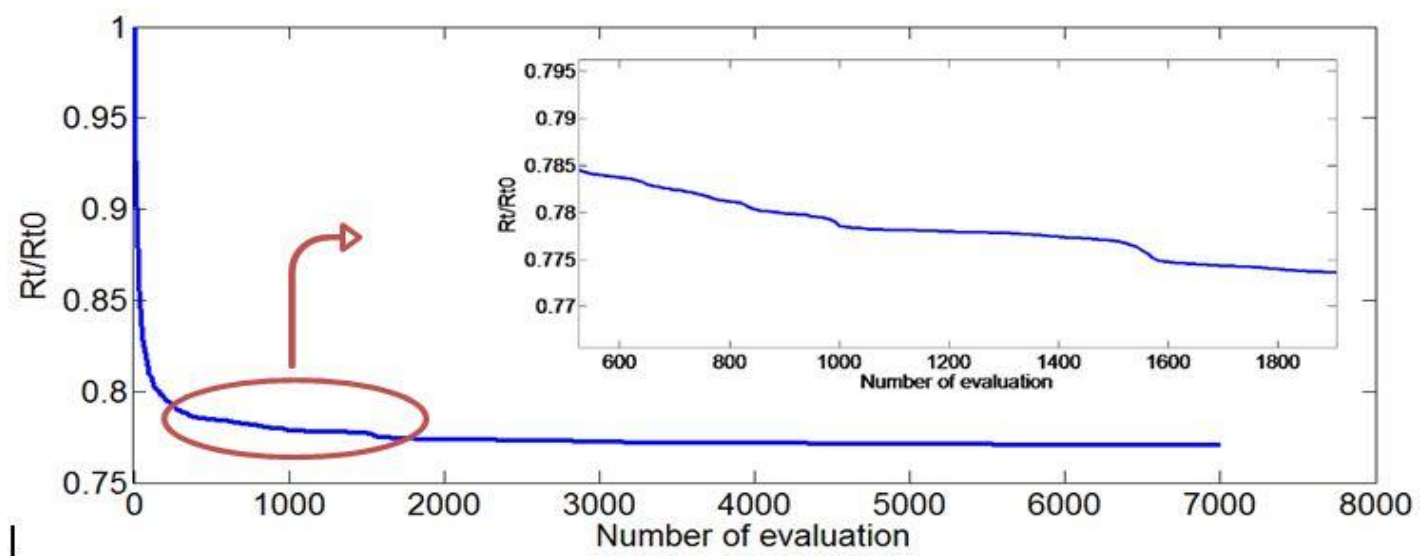

c) total resistance

Figure 9: The history of the resistance of the $\mathrm{S} 60$ hull by evaluation number

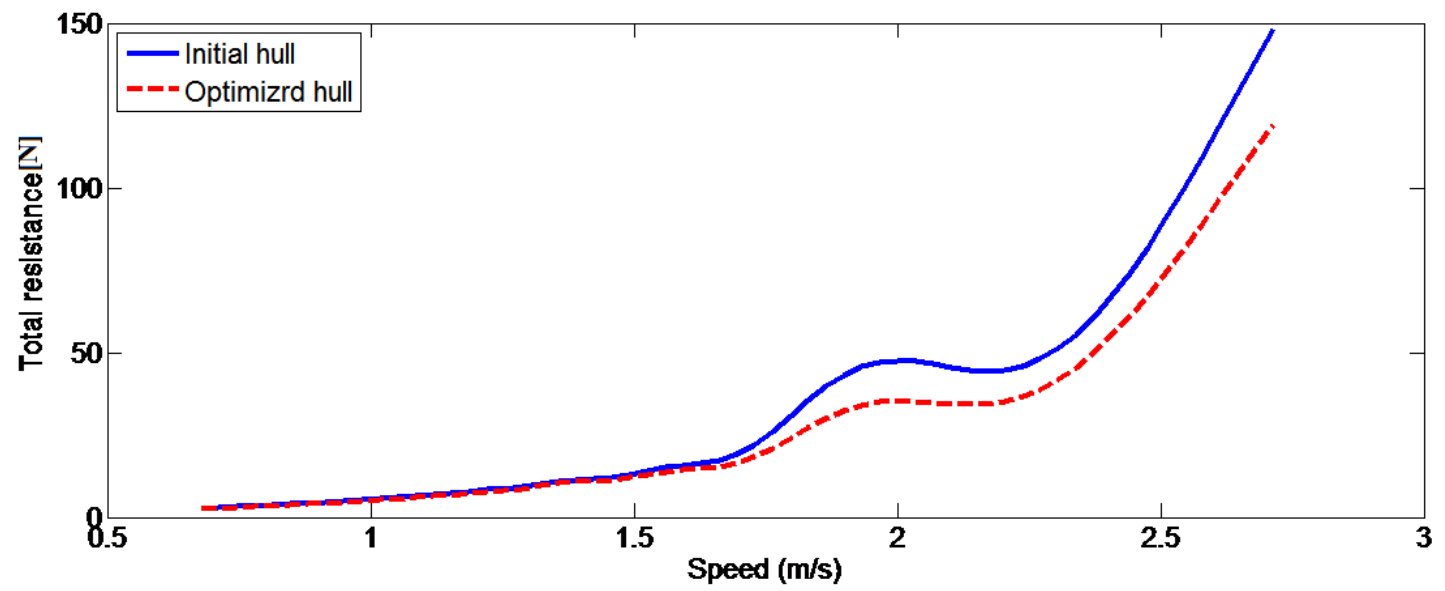

Figure 10: The total resistance for the initial S60 hull and the optimized hull

Figure 9 demonstrates the changes of the frictional, wave and total resistances of hull by evaluation number. As can be seen in this figure, the frictional resistance initiates high and decreases very rapidly. This is due to changes in the length and the wetted surface of the hull as in the previous case. The variation of wetted surface of the hull influences the frictional resistance and other resistance components. Also, it is indicated that in the early phase of the optimization process the wave resistance is lowered rapidly, and becomes very small compared to the frictional resistance and then is maintained in the remaining of the evolution procedure. 
Evaluating the hydrodynamic performance of the initial hull and the optimized hull in terms of the total resistance is shown in Figure 10. As can be seen in this figure, although the hull form has been optimized for a single speed $(V=2.14)$, the optimal hull form has less total resistances over the broad range of the speed, especially where the optimization technique is run. The optimization process for this case takes around 123 minutes.

In both cases, it is interesting to note that although the width of the models have risen, the draft and wetted surface of the models have lowered significantly and thus the total resistance of the optimized hulls have become smaller than that of the initial hulls.

\section{Conclusion}

A numerical method has been proposed for hydrodynamic hull form optimization in calm water with respect to total resistance as the only objective function. The evolution strategy algorithm is combined with a numerical method for minimizing total resistance characteristic (the wave making resistance based on Michell's thin ship theory and the viscous resistance based on ITTC 1957 and an experimental formula). The design variables are included the hull offsets and the main dimensions (Length, breadth and draft) and the displacement is used as the design constraint during the optimization at constant speed Fn is 0.316 with Wigley and S60 hull forms as standard models to develop optimized ship hull forms. Compared with the initial hull the total resistance of the improved hull is reduced by $18.2 \%$ in the first example and $22.98 \%$ in the second one. The hull fairing procedure has been applied by using NURBS. As can be showed in figures, the reduction percent achieved especially in wave resistances is considerable comparing with other papers. The gains in terms of total resistance reductions were considerable at both cases and this resulted in improvements in the entire ship range. Therefore, we can conclude that evolution strategies used in this study are effective and robust techniques for hull form optimization.

\section{Acknowledgment}

Authors are grateful to the marine research center (MRC) of AUT for some financial support.

\section{References}

Back, T. and Schwefel, H. P. (1993): An overview of evolutionary algorithms for parameter optimization, Journal of Evolutionary Computation, vol. 1, no. 1, pp. 1-23. http://dx.doi.org/10.1162/evco.1993.1.1.1

Back, T., Fogel, D. B. and Michalewicz, E. Z. (2000): Evolutionary Computation 1: Basic Algorithms and Operators, Bristol (UK), Institute of Physics. http://dx.doi.org/10.1887/0750306653

Beyer, H. G. and Schwefel, H. P. (2002): Evolution Strategies: A Comprehensive Introduction, Journal of Natural Computing, vol. 1, no. 1, pp. 3-52. http://dx.doi.org/10.1023/A:1015059928466

Biliotti, I., Brizzolara, S., Viviani, M., Vernengo, G., Ruscelli, D., Galliussi, M., Guadalupi, D. and Manfredini, A. (2011): Automatic Parametric Hull Form Optimization of Fast Naval Vessels, 11th International Conference on Fast Sea Transportation (FAST), Honolulu, Hawaii, USA.

Davis, P. J. and Rabinowitz, P. (1984): Methods of Numerical Integration, 2nd ed., Academic Press New York. Day, A. H. and Doctors, L.J. (2001): Rapid estimation of near-and far-field wave wake from ships and application to hull-form design and optimization, Journal of Ship Research, vol. 45, no. 1, pp. 73-84.

De, A., Kumar A., (2006): "Opti-Marine-Ware" (Optimization of Vessel's Parameters through Spreadsheet Model), J. of Naval Arch \& Marine Eng., Vol 3, No. 2, pp.49-58.

Eiben, A. E. and Smith, J. E. (2003): Introduction to Evolutionary Computing, 1st ed., Springer, Natural Computing Series.

Gammon, M. A. (2011): Optimization of fishing vessels using a Multi-Objective Genetic Algorithm, Journal of Ocean Engineering, vol. 38, no. 10, pp. 1054-1064. http://dx.doi.org/10.1016/j.oceaneng.2011.03.001

Gotman, A. Sh. (2002): Study of Michell's Integral and Influence of Viscosity and Ship Hull Form on Wave Resistance, Oceanic Engineering International, Vol. 6, No. 2, pp. 74-115.

Grigoropoulos, G. J., Chalkias, D. S. and Tikkos, C. (2004): Multi-objective hull form optimization of highspeed vessels, Intl. conf. HIPER. PMCid:1772407 
Grigoropoulos, G. J. and Chalkias, D. S. (2010): Hull-form optimization in calm and rough water, Journal of Computer-Aided Design, vol. 42, no. 11, pp. 977-984. http://dx.doi.org/10.1016/j.cad.2009.11.004

Han, S., Lee, Y. S., Choi, Y. B. (2012): Hydrodynamic hull form optimization using parametric models, J Mar Sci Technol, vol. 17, no. 1, pp. 1-17. http://dx.doi.org/10.1007/s00773-011-0148-8

Insel, M. (1990): An investigation into the drag components of high speed catamaran, Ph.D Thesis, Department of ship science, University of Southampton, UK.

Ju, S. (1983): Study of total and viscous drag for the Wigley parabolic ship form, IIHR Report, no. 261, p. 35.

Jun, A. and Kuniharu, N. (2004): A Trial to Reduce Wave making Resistance of Catamaran-Hull Form Improvement Using Real-Coded Genetic Algorithm, Transactions of the West-Japan Society of Naval Architects, vol. 107, pp.1-13.

Karim, M. M., Suzuki, K. and Kai H. (2004): Optimal design of hydrofoil and marine propeller using microgenetic algorithm (GA), Journal of Naval Architecture \& Marine Engineering, Vol. 1, No. 1, pp.41-61.

Kim, H., Yang, C., Löhner, R. and Noblesse, F. (2008): A Practical Hydrodynamic Optimization Tool for the Design of a Monohull Ship, in Proc. ISOPE Conf., Vancouver, Canada.

Kim, H., Yang, C., Kim, H., Chun, H. (2009): Hydrodynamic Optimization of a Modern Container Ship using Variable Fidelity Models, Proc. ISOPE Conf., Osaka, Japan.

Kim, H., Yang, C., Kim, H., Chun, H. (2012): Hydrodynamic Optimization of Ship Hull Form Using Finite Element Method, and Variable Fidelity Models, Proc. ISOPE Conf., Rhodes, Greece.

Percival, S., Hendrix, D., and Noblesse, F. (2001): Hydrodynamic optimization of ship hull forms, Journal of Applied Ocean Research, vol. 23, no. 6, pp. 337-355.

Piegl, L. and Tiller, W. (1995): The NURBS Book, Springer-Verlag. http://dx.doi.org/10.1007/978-3-64297385-7

Rechenberg, I. (1973): Evolution strategie: Optimierung Technisher Systemenach Prinzipien des Biologischen Evolution, Fromman- Hozlboog Verlag, Stuttgart.

Saha, G. K., Suzuki, K. and Kai, H. (2004): Hydrodynamic optimization of ship hull forms in shallow water, Journal of Marine Science and Technology, vol. 9, no.2, pp.51-62. http://dx.doi.org/10.1007/s00773-003-0173-3 Schwefel, H. P. (1995): Evolution and Optimum Seeking, Wiley Interscience, New York. PMid:8847117

Tuck, E. O. and Lazauskas, L. (1998): Optimum hull spacing of a family of multihulls, Dept. Applied Mathematics Technical Report, The University of Adelaide, Adelaide, Australia.

Tuck, E. O. and Lazauskas, L. (2008): Drag on a ship and Michell's integral, ICTAM 2008, Adelaide, South Australia.

Tuck, E. O., Scullen, D. C. and Lazauskas, L. (2002): Wave patterns and minimum wave drag for high-speed vessels, in Proc. of 24th Symposium on Naval Hydrodynamics, Fukuoka, Japan.

Zhang, B. J. (2009): The optimization of the hull form with the minimum wave making resistance based on Rankine source method, Journal of Hydrodynamics, vol. 21, no. 2, pp. 277-284. http://dx.doi.org/10.1016/S1001-6058(08)60146-8

Zhang, B. J. (2012): Research on optimization of hull lines for minimum resistance based on Rankine source method, Journal of Marine Science and Technology, vol. 20, no. 1, pp. 89-94.

Vyselaar, D., Klaptocz, V. and Calisal, S. M. (2007): A numerical approach to resistance reduction of displacement vessels using parabolic waterlines, Ships and Offshore Structures, vol. 2, no. 2, pp.127-136. http://dx.doi.org/10.1080/17445300701430176 